\title{
Surface plasmon resonance for characterization of large-area atomic-layer graphene film: supplementary
} material

\author{
Henri Jussila, ${ }^{1,}{ }^{*}$ He Yang, ${ }^{1}$ Niko Granqvist, ${ }^{2}$ and Zhipel Sun ${ }^{1, *}$ \\ ${ }^{1}$ Department of Micro- and Nanosciences, Aalto University, Tietotie 3, Fl-00076 Espoo, Finland \\ ${ }^{2}$ BioNavis Ltd. Elopellontie 3C, 33470 Ylöjärvi, Finland \\ *Corresponding authors: henri.jussila@aalto.fi; zhipei.sun@aalto.fi \\ Published 5 February 2016
}

This document provides supplementary information to "Surface plasmon resonance for characterization of largearea atomic-layer graphene film," http://dx.doi.org/10.1364/optica.3.000151. @ 2016 Optical Society of America http://dx.doi.org/10.1364/optica.3.000151.s001

\section{Parameters used for the model of the SPR sensors}

The adhesion layer is Chromium in our SPR sensors. The parameters and the complex refractive indices of different materials in the SPR sensors are listed in Table S1. These values were used to calculate the SPR spectra of the graphene coated SPR sensors by fitting the refractive index and the thickness of the transferred graphene layers so that the calculated spectrum best matches with the measured one. Note that some of the values listed in Table S1 may differ from these used in the literature. However, these values have been obtained empirically from the best fits of the measured SPR spectra of the used uncoated sensors, and therefore, their usage is more than justified.

\section{Repeatability of our SPR measurements}

The repeatability of the SPR measurements was verified by measuring the SPR spectrum from different positions of the fabricated SPR sensors. General trend seen in these measurements was that the variation of the SPR angle was smaller than $0.01^{\circ}$. Therefore, the scattering of the measured properties within the sample was small which is well expected because SPR measurement gathers information from the large area $\left(\sim \mathrm{mm}^{2}\right)$, and thus, reveals information from the average properties of the fabricated graphene samples.

To estimate the scattering between different graphene samples, another $\mathrm{Au} / \mathrm{Al}_{2} \mathrm{O}_{3} \mathrm{SPR}$ sensor was prepared. Figure $\mathrm{S} 1$ shows SPR spectrum measured prior and after the graphene transfer from this sensor. Note that the graphene layer transferred on this sensor originated from a different CVD growth run. As observed, the sensor SPR angle is at $44.64^{\circ}$ for uncoated sensor while for the sensor coated with $1 \mathrm{~L}$ graphene it is at $44.98^{\circ}$. This gives SPR angle change of $\sim 0.34^{\circ}$ (corresponding to about $1^{\circ} / \mathrm{nm}$ ), which agrees extremely well with the results (Fig. 3a) presented in the main document.

Table S1: Parameters Used for the Model of Our SPR Sensors.

\begin{tabular}{|l|l|l|l|l|l|}
\hline Material & \multirow{2}{*}{$\begin{array}{l}\text { Thickness } \\
(\mathrm{nm})\end{array}$} & \multicolumn{2}{|l|}{$670 \mathrm{~nm}$} & \multicolumn{2}{l|}{$785 \mathrm{~nm}$} \\
\cline { 3 - 6 } & & $\mathrm{n}$ & $\mathrm{k}$ & $\mathrm{n}$ & $\mathrm{k}$ \\
\hline $\mathrm{Air}$ & $\infty$ & 1.0003 & 0.0000 & 1.0003 & 0.0000 \\
$\mathrm{Al}_{2} \mathrm{O}_{3}$ & 10 & 2.2510 & 0.1555 & 2.4372 & 0.1670 \\
$\mathrm{Au}$ & 45 & 0.2599 & 4.2529 & 0.2936 & 5.2910 \\
$\mathrm{Cr}$ & 0.5 & 2.6262 & 1.0000 & 1.0000 & 3.2211 \\
Glass & $\infty$ & 1.5202 & 0.0000 & 1.5162 & 0.0000 \\
\hline
\end{tabular}

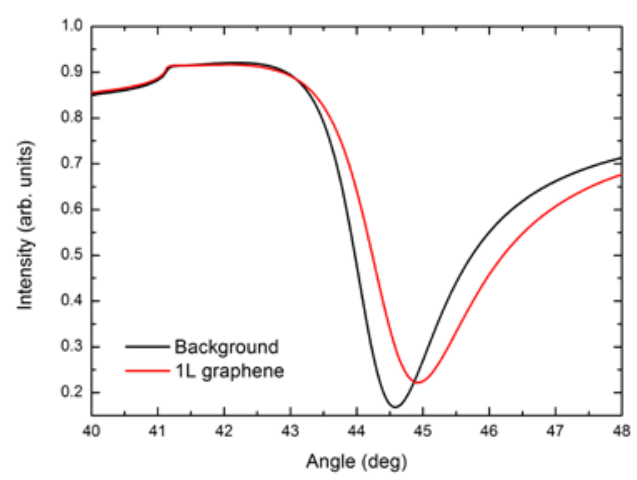

Figure S1: SPR curves measured from another graphene coated $\mathrm{Au} / \mathrm{Al}_{2} \mathrm{O}_{3} \mathrm{SPR}$ sensor. 